\title{
Capillary-Channeled Polymer (C-CP) Fibers as a Stationary Phase for Sample Clean-Up of Protein Solutions for Matrix-Assisted Laser/ Desorption Ionization Mass Spectrometry
}

\author{
Benjamin T. Manard, R. Kenneth Marcus \\ Department of Chemistry, Biosystems Research Complex, Clemson University, Clemson, SC 29633, USA
}

\begin{abstract}
Capillary-channeled polymer (C-CP) fibers are employed in a micropipette tip format to affect a stationary phase for the solid phase extraction (SPE) of proteins from buffer solutions prior to MALDI-MS analysis. Proteins readily adsorb to the polypropylene (PP) C-CP fibers while buffer species are easily washed off the tips using $\mathrm{DI}-\mathrm{H}_{2} \mathrm{O}$. Elution of the solutes is achieved with an aliquot of 50:50 ACN: $\mathrm{H}_{2} \mathrm{O}$, which is compatible with the subsequent spotting on the MALDI target with the matrix solution. Lysozyme and cytochrome $c$ are used as test species, with a primary buffer composition of $100 \mathrm{mM}$ Tris- $\mathrm{HCl}$. In this case, direct MALDI-MS produces no discernible protein signals. SPE on the C-CP fibers yields high fidelity mass spectra for $1 \mu \mathrm{L}$ sample volumes. Limits of detection for cytochrome $c$ in $100 \mathrm{mM}$ Tris- $\mathrm{HCl}$ are on the order of $40 \mathrm{nM}$. Extraction of cytochrome $c$ from buffer concentrations of up to $1 \mathrm{M} \mathrm{Tris-} \mathrm{HCl}$, provides signal recoveries that are suppressed by only $\sim 50 \%$ versus neat protein solutions. Finally, extraction of $3.1 \mu \mathrm{M}$ cytochrome $c$ from a synthetic urine matrix exhibits excellent recovery.
\end{abstract}

Key words: Capillary-channeled polymer, Fiber, SPE, MALDI-MS, Proteins

\section{Introduction}

$\mathrm{P}$ roteomics is centered on the ability to identify and characterize proteins while maintaining their structural integrity. With this in mind, proteolysis and cell digestions must be performed in buffered solutions to ensure stability, preserve biological function, or to maintain nontoxic cellular surroundings. Unfortunately, buffers are detrimental in protein analysis by mass spectrometry because they suppress the signal from the desired proteins [1]. Matrix-assisted laser desorption/ionization-mass spectrometry (MALDI-MS) is a widely used technique for the analysis of biomacromolecules because of its ability to softly-ionize analytes with little or no fragmentation; however, studies have shown samples containing buffer concentrations of $100 \mathrm{mM}$ or greater must be desalted prior to MALDI-MS [2, 3]. Because of the

Correspondence to: R. Marcus; e-mail: marcusr@clemson.edu importance of the problem of buffer/matrix suppression, various approaches have been explored to remove these contaminants prior to MALDI-MS [1-5].

High-performance liquid chromatography (HPLC), 2-D electrophoresis, and centrifugation are common techniques employed for the clean-up of protein samples prior to MALDIMS [1]. In the absence of needing a chemical separation of proteins, solid phase extraction (SPE) is the most popular technique for clean-up of biological samples. SPE works by adsorbing target molecules onto the surface of the stationary phase while buffers and surfactants are washed away [6]. Proteins are eluted from the stationary phase in a solvent that is generally compatible with the direct deposition on the MALDI target. Primary advantages of SPE over other techniques include: reduced analysis time, small amounts of elution solvent, and small volumes of (typically) low-cost sorbent materials [7]. More recently, SPE platforms have been miniaturized and used with micropipette tip-based fluid 
manipulations for protein desalting, allowing effective handling of microliter-volume samples [8,9]. Many approaches use a $\mathrm{C}_{18}$ derivatized porous silica media for the purification of peptides from buffers/salts as described above [10]. Problems encountered in micropipette tip platforms include robustness and sensitivity issues for small amounts of protein because of inefficient interactions and low recoveries [11].

This laboratory has developed capillary-channeled polymer (C-CP) fiber stationary phases for the liquid chromatography separation and solid phase extraction (SPE) of proteins [1215]. C-CP fibers are advantageous when compared to other stationary phases because of the wide range of polymer/surface chemistries available, robustness, low cost, and the ability for microanalytical separations. A subtle, but very important, characteristic is the lack of porosity of the C-CP fibers, resulting in rapid adsorption/desorption kinetics and high recoveries [13]. In addition to chromatographic columns, polypropylene (PP) C-CP fibers housed in micropipette tips have been employed for the SPE/desalting of protein solutions prior to ESI-MS [15]. Here, proteins adsorb onto the hydrophobic PP C-CP fiber surfaces while allowing salts to directly pass or be washed off with a simple aqueous rinse step. Bound proteins are eluted with ESI-friendly solvents, such as acetonitrile:water $\left(\mathrm{ACN}: \mathrm{H}_{2} \mathrm{O}\right)$ mixtures. Intensity, signal-tonoise ratios $(\mathrm{S} / \mathrm{N})$, and spectral clarity of analytes were improved dramatically in $150 \mathrm{mM}$ buffer due to the clean-up with C-CP fibers prior to ESI-MS analysis.

Polypropylene C-CP fibers are employed here as a stationary phase for SPE of proteins from buffer solutions prior to MALDI-MS analysis. A new protocol for the SPE processing, employing a simple centrifugation method, provides far greater sample throughput and overall superior precision than manual aspiration $[15,16]$. Efficient desalting is performed for proteins (cytochrome $c$ and lysozyme) on the sub-micromolar level (nanogram absolute mass) in the presence of up to $1 \mathrm{M}$ Tris $-\mathrm{HCl}$. The practicality of the method is demonstrated by the successful desalting of single-micromolar concentrations of protein from a mockurine sample. It is believed that $\mathrm{C}-\mathrm{CP}$ fibers in a micropipette tip format present a very promising approach to protein sample clean-up prior to MALDI-MS analysis.

\section{Experimental}

\section{Reagents and Chemicals}

Cytochrome $c$ (bovine heart, MW=12.2 kDa), lysozyme (chicken egg white, MW=14.3), and sinapinic acid (SA) were obtained from Sigma-Aldrich (Milwaukee, WI, USA). Tris-HCl was from Teknova, (Hollister, CA, USA) and ACS grade acetonitrile (ACN) was obtained from Fisher Scientific (Pittsburg, PA, USA). MilliQ water $(18.2 \mathrm{M} \Omega / \mathrm{cm})$ derived from a NANOpure Diamond Barnstead/Thermolyne Water System (Dubuque, IA, USA) was used in preparation of buffer and proteins solutions, and aqueous mobile phases. A synthetic urine matrix was prepared using the typical constituent concentrations of $233 \mathrm{mM} \mathrm{KHCO} 3,110 \mathrm{mM}$ $\mathrm{KCl}, 13 \mathrm{mM} \mathrm{K}_{2} \mathrm{SO}_{4}, 65 \mathrm{mM}$ glycine, and $5 \mathrm{mM}$ urea.

\section{Fiber-Tip Construction}

C-CP SPE tip preparation methodology is identical to the construction of microbore C-CP fiber HPLC columns [14]. The $\mathrm{C}-\mathrm{CP}$ fibers are pulled through $0.8-\mathrm{mm}$ i.d. fluorinated ethylene polypropylene (FEP) tubing (Cole Parmer, Vernon Hills, IL, USA). In the present study, a total of 658 PP fibers of $\sim 55 \mu \mathrm{m}$ diameter were pulled collinearly through $\sim 300 \mathrm{~mm}$ of the tubing, yielding an interstitial (void) fraction of $\varepsilon_{\mathrm{i}} \approx 0.6$. Approximately $10 \mathrm{~mm}$ was cut off an end of the packed tubing with a surgical grade scalpel so that the fiber and tubing ends were flush. On the opposite end of the tubing, a $\sim 6 \mathrm{~mm}$ free space was left between the tubing and the fiber ends. The end of the tubing with the dead space was slipped over a $1 \mathrm{~mL}$ micropipette tip (Redi-Tip; Fisher Scientific, Pittsburg, PA, USA). The tubing was then cut so that $1 \mathrm{~cm}$ of packed fibers was attached to each tip. The tip construction process yielded $\sim 20$ tips from the $\mathrm{C}-\mathrm{CP}$ fiber column.

\section{Adaptation to the Centrifugation Format}

A centrifugation-based approach as been implemented, resulting in higher sample throughput through parallel processing and greater levels of experimental control [16]. A pipette tip adapter was made so the fiber-packed tips could be placed into a six-bucket benchtop centrifuge (Clinical 50; VWR, West Chester, PA, USA). The conical portion $(\sim 10 \mathrm{~mm})$ of a $1.7 \mathrm{~mL}$ microtube (Genesee Scientific, San Diego, CA, USA) was cut perpendicular to the tube axis. The fiber-packed micropipette tip was placed inside the top of the microtube. The microtube was then placed into a $15 \mathrm{~mL}$ conical centrifuge tube (VWR) as depicted in Fig. 1, for placement in the centrifuge.

\section{Protein Loading, Washing, and Elution}

Solutions of cytochrome $c$ were made in Tris- $\mathrm{HCl}$ buffer with buffer concentrations of (100-1000 mM). A $2.5 \mu$ M lysozyme solution was also made in $100 \mathrm{mM}$ Tris-HCl buffer. Loading aliquots $(3-10 \mu \mathrm{L})$ of the protein solutions were centrifuged through the fiber tips at 4000 RPM for $2 \mathrm{~min}$. The washing step included a $20 \mu \mathrm{L}$ aliquot of MilliQ water, centrifuged at 4000 RPM for $2 \mathrm{~min}$. The proteins were eluted from the fiber surfaces by centrifuging an aliquot equal to the loading volume of 50:50 ACN: $\mathrm{H}_{2} \mathrm{O}$ through the tips at $4000 \mathrm{RPM}$ for $2 \mathrm{~min}$. The total processing time for the procedure (for multiple tips in parallel) is approximately $10 \mathrm{~min}$.

\section{Mass Spectrometry}

A Bruker Daltonics (Billerica, MA, USA) Microflex LRF, MALDI-TOF mass spectrometer was used in the positive ion, linear mode for the protein analysis. The Bruker 


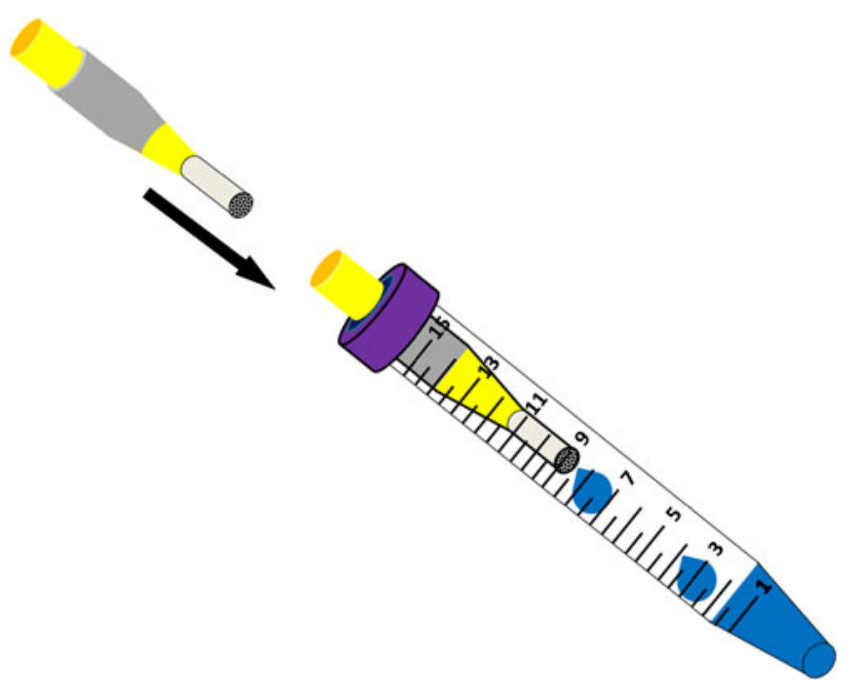

Figure 1. Adaptation of the C-CP fiber-packed tips format for centrifugation-driven SPE. The bottom of the microtube is removed and the $\mathrm{C}-\mathrm{CP}$ fiber-packed tip is inserted. The microtube with the fiber tip is then inserted into a $15 \mathrm{~mL}$ centrifuge tube so that it can be place into a centrifuge device. The fractions are then collected in separate centrifuge tubes

Compass software was used for control and data processing. The MALDI-TOF is equipped with a nitrogen laser $(337 \mathrm{~nm})$ operating at a pulse rate of $60 \mathrm{~Hz} .200$ laser shots at $79 \%$ laser power were used to acquire the spectra. A $20 \mathrm{mg} \mathrm{mL} \mathrm{m}^{-1}$ solution of sinapinic acid in 50:50 ACN: $\mathrm{H}_{2} 0$ with $0.1 \%$ TFA was used as the matrix solution. The MALDI target was prepared by spotting $1 \mu \mathrm{L}$ of the matrix solution followed by $1 \mu \mathrm{L}$ of the protein solutions, with time for drying between each step. Signal-to-noise ratios are based on the standard deviation of the signals in a 100 Da mass window centered $2000 \mathrm{Da}$ below the protein molecular ions.

\section{Results and Discussion}

\section{MALDI-MS of Cytochrome c and Lysozyme from $100 \mathrm{mM}$ Tris Buffer}

The SPE of protein solutions was assessed by comparing analyte signal intensities and signal-to-noise ratios of protein samples before and after extractions with the C-CP fiber micropipette tips. Separate $10 \mu \mathrm{L}$ aliquots of each protein were loaded onto the $\mathrm{C}-\mathrm{CP}$ fiber tips. The mass spectra presented in Fig. 2 illustrate the relationship between the signals of $3.1 \mu \mathrm{M}$ cytochrome $c$ and $2.8 \mu \mathrm{M}$ lysozyme in $100 \mathrm{mM}$ Tris buffer before and after SPE desalting. The mass spectra show no discernible signals representative of the proteins in the presence of $100 \mathrm{mM}$ Tris buffer. On the other hand, proteins extracted from the buffer using the C$\mathrm{CP}$ fibers yielded high fidelity spectra. Comparisons with the protein signal intensities for the SPE extracted proteins to neat protein solutions (same solvent and concentration,
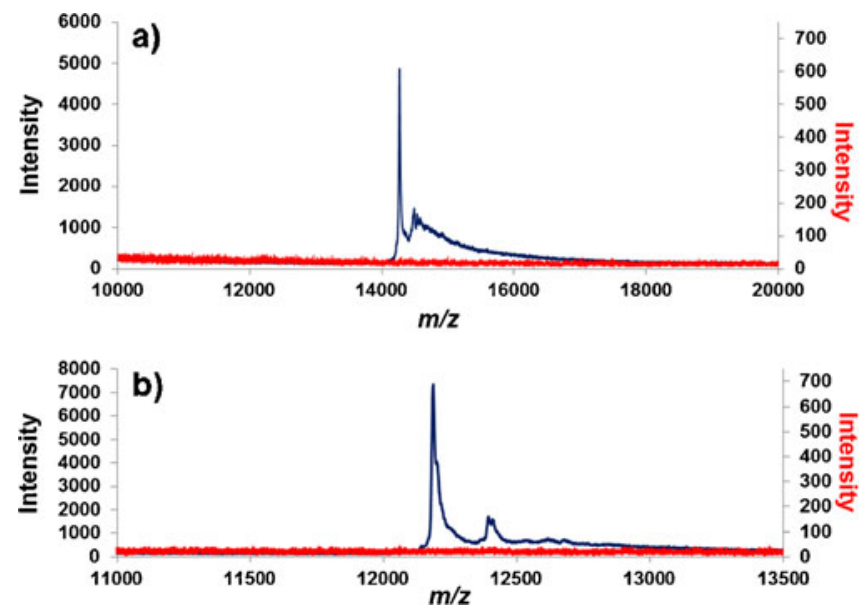

Figure 2. MALDI-MS spectra of (top) $2.8 \mu \mathrm{M}$ lysozyme and (bottom) $3.1 \mu \mathrm{M}$ cytochrome $c$ in $100 \mathrm{mM}$ Tris buffer before (in red) and after (in blue) extraction on PP C-CP fiber-based micropipette tips

without buffer) provides a level of assessment of the degree of overall recovery. There is a $<5 \%$ loss in signal intensity through the course of the SPE process, perhaps due to irreversible binding to the fibers or the micropipette tip components themselves. In any case, such performance is testament to the overall efficiency of the methodology.

Another important aspect in proteomic applications is the ability to clean up samples having low concentrations of protein in the presence of high matrix concentrations. A serial dilution $(0.25-3.1 \mu \mathrm{M})$ of cytochrome $c$ in the $100 \mathrm{mM}$ Tris buffer was prepared and analyzed in triplicate. A $1 \mu \mathrm{L}$ volume of the lowest protein concentration (representing a protein mass of $3 \mathrm{ng}$ ) was still detected with spectral clarity. Extrapolation to a limit of detection based on an $\mathrm{S} / \mathrm{N}=3$ yields a value of $\sim 0.5 \mathrm{ng}$, or $\sim 40 \mathrm{nM}$ in the original buffer solution. This ability to perform buffer removal on nanogram quantities of protein in a high abundance of buffer (buffer:protein ratio of 400,000:1) illustrates the effectiveness of the process.

\section{Effect of Tris Buffer Concentration on MALDI-MS Recoveries}

The concentration of buffers employed can differ substantially in bioanalysis applications, with varying effects in any MS analysis. Additionally, salt/matrix concentrations vary tremendously across sample types, such as blood, cell extracts, or urine. With this in mind, desalting techniques should be effective at not only buffer concentrations $(100 \mathrm{smM})$, but perhaps concentrations up to $1 \mathrm{M}$. Solutions of $3.1 \mu \mathrm{M}$ cytochrome $c$ were prepared in buffer concentrations ranging from $100 \mathrm{mM}$ to $1 \mathrm{M}$ (including a neat solution), to quantify the overall signal recoveries as well as the resultant $\mathrm{S} / \mathrm{N}$ characteristics. Both quantities decrease monotonically as the buffer concentration increases, but exhibiting only $50 \%-60 \%$ depressions versus the neat protein. Important to note here is 

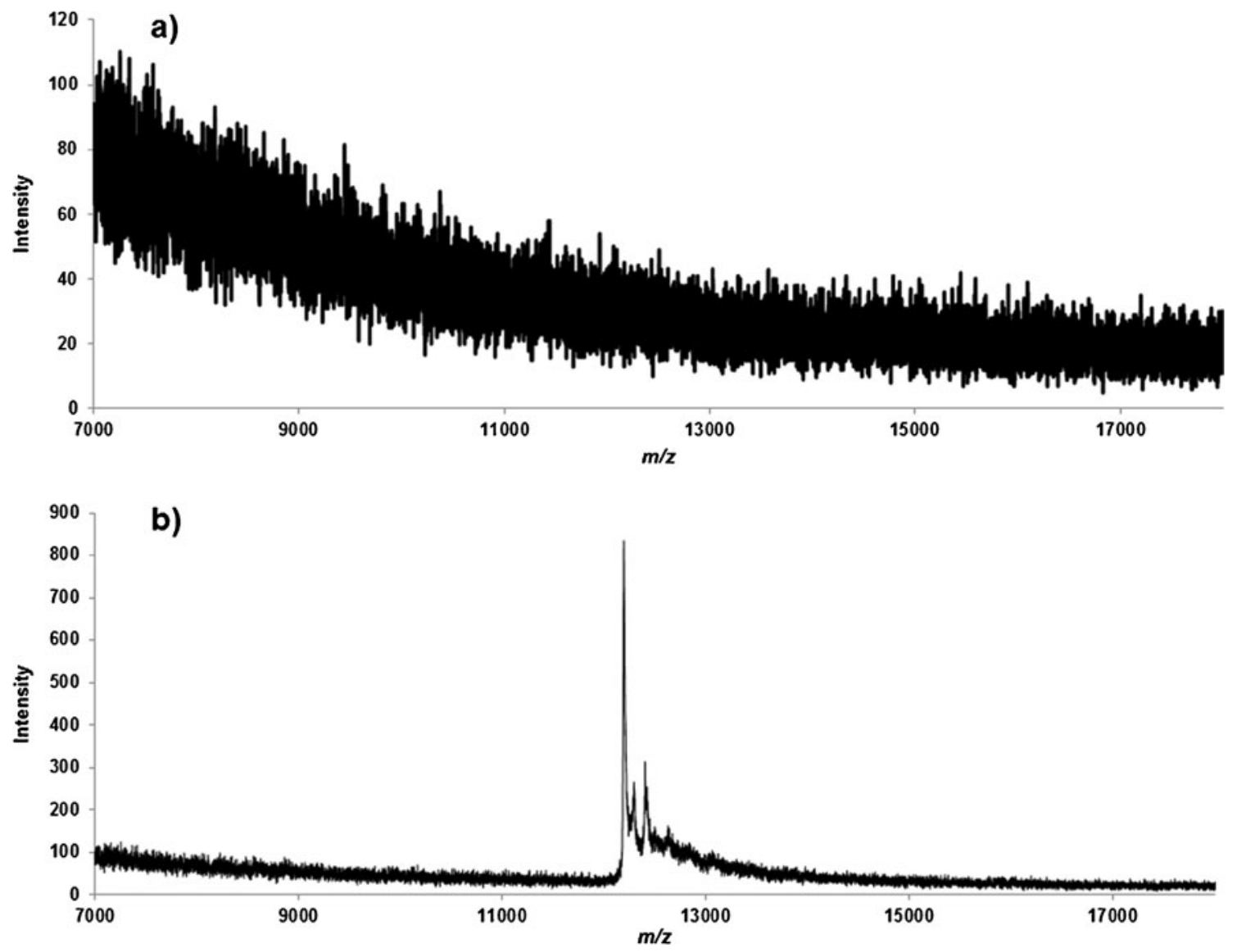

Figure 3. MALDI-MS spectra of (a) $3.1 \mu \mathrm{M}$ cytochrome in synthetic urine and (b) $3.1 \mu \mathrm{M}$ cytochrome in synthetic urine following SPE on PP C-CP fiber based micropipette tips

that each of the samples was subjected to the same wash/elute procedure. So the results obtained may be improved further by simple use of a higher-volume $\mathrm{DI}-\mathrm{H}_{2} \mathrm{O}$ washing procedure.

The relative immunity to high salt/buffer concentrations begs the question as to the direct applicability of the C-CP fiber SPE method. To test this point, cytochrome $c$ was spiked into a synthetic urine matrix at a concentration of $3.1 \mu \mathrm{M}$ and analyzed directly by MALDI-MS. As seen in Fig. 3a, the cytochrome $c$ molecular ion is not at all detectable at the $3.1 \mu \mathrm{M}$ level in the urine matrix. Following extraction on the C-CP fiber tips, the cytochrome $c$ was detected with high spectral clarity as depicted in Fig. 3b, illustrating the effectiveness of protein extraction from a urine matrix. Consistent with the results described previously, the LOD for the protein in the mock urine is $\sim 67 \mathrm{nM}$ for this $1 \mu \mathrm{L}$ sampling.

\section{Conclusion}

The results from these experiments extend the potential of PP C-CP fibers as stationary phase for SPE methods for sample clean-up prior to MALDI-MS analysis. C-CP fiber tips effectively retain protein and allow weakly bound organics and buffers to be washed away, with the proteins eluted in MALDI-friendly solvents. This purification procedure improves analyte signal response and $\mathrm{S} / \mathrm{N}$ characteristics, which are critical for biomolecule analysis. The ability to perform SPE for samples across these matrix conditions supports the potential use of the C-CP fiber SPE tips for various biofluids (e.g., blood, saliva) prior to MALDI-MS. Optimization of several parameters including fiber packing density, tip length, volumes of washing and elution steps, and elution solvents are under investigation with the potential to improve the capabilities demonstrated here.

\section{Acknowledgment}

The authors acknowledge that this material is based upon work supported by the National Science Foundation Division of Chemistry under grant no. 1011820 (co-funded by the MPS/ CHE, ENG/CBET, and EPSCoR).

\section{References}

1. Mann, M., Hendrickson R. C., Pandey A.: Analysis of proteins and proteomes by mass spectrometry. Annu. Rev. Biochem. 70, 437-473 (2001)

2. Zhang, L., Orlando R.: Solid-phase extraction/MALDI-MS: Extended ion-pairing surfaces for the on-target cleanup of protein samples. Anal. Chem. 71(20), 4753-4757 (1999) 
3. Xu, Y. D., Bruening M. L., Watson J. T.: Nonspecific, on-probe cleanup methods for MALDI-MS samples. Mass Spectrom. Rev. 22(6), 429-440 (2003)

4. Chin, E. T., Papac D. I.: The use of a porous graphitic carbon column for desalting hydrophilic peptides prior to matrix-assisted laser desorption/ionization time-of-flight mass spectrometry. Anal. Biochem. 273(2), 179-85 (1999)

5. Warren, M. E., Brockman A. H., Orlando R.: On-probe solid-phase extraction/MALDI-MS using ion-pairing interactions for the cleanup of peptides and proteins. Anal. Chem. 70(18), 3757-61 (1998)

6. Majors, R. E.: The role of polymers in solid-phase extraction and sample preparation. LC-GC North Am 26(11), 1074-1090 (2008)

7. Huck, C. W., Bonn G. K.: Recent developments in polymer-based sorbents for solid-phase extraction. J. Chromatogr. A 885(1/2), 51-72 (2000)

8. Rappsilber, J., Ishihama Y., Mann M.: Stop and go extraction tips for matrix-assisted laser desorption/ionization, nanoelectrospray, and LC/MS sample pretreatment in proteomics. Anal. Chem. 75(3), 663-70 (2003)

9. Shukla, A., Majors R. E.: Micropipette tip-based sample preparation for bioanalysis. LC-GC North America 23(7), 646-660 (2005)

10. Baczek, T.: Fractionation of peptides in proteomics with the use of pIbased approach and ZipTip pipette tips. J. Pharma. Biomed. Anal. 34 (5), 851-860 (2004)
11. Ishihama, Y., Rappsilber J., Mann M.: Modular stop and go extraction tips with stacked disks for parallel and multidimensional peptide fractionation in proteomics. J. Proteome Res. 5(4), 988-994 (2006)

12. Marcus, R. K., Davis W. C., Knippel B. C., LaMotte L., Hill T. A., Perahia D., Jenkins J. D.: Capillary-channeled polymer fibers as stationary phases in liquid chromatography separations. J. Chromatogr. A 986(1), 17-31 (2003)

13. Nelson, D. M., Marcus R. K.: Characterization of capillary-channeled polymer fiber stationary phases for high-performance liquid chromatography protein separations: comparative analysis with a packed-bed column. Anal. Chem. 78(24) 8462-8471 (2006)

14. Stanelle, R. D., Mignanelli M., Brown P., Marcus R. K.: Capillarychanneled polymer (C-CP) fibers as a stationary phase in microbore high-performance liquid chromatography columns. Anal. Bioanal. Chem. 384(1), 250-258 (2006)

15. Fornea, D. S., Wu Y., Marcus R. K.: Capillary-channeled polymer fibers as a stationary phase for desalting of protein solutions for electrospray ionization mass spectrometry analysis. Anal. Chem. 78(15), 5617-5621 (2006)

16. Pittman, J. J., Klep V., Luzinov I., Marcus R. K.: Extraction of metals from aqueous systems employing capillary-channeled polymer (C-CP) fibers modified with poly(acrylic acid) (PAA). Anal. Methods 2(5), 461-469 (2010) 